Japan. J. Math.

Vol. 16, No. 2, 1990

\title{
The strict domain of attraction of strictly stable law with index 1
}

\author{
By Takaaki Shimura
}

(Received October 17, 1989)

\section{§1. Introduction}

The set of strictly stable distributions is a proper subset of the set of stable distributions. If the index is not 1 , then every stable distribution is a shift of a strictly stable distribution (prescribed in Section 2). While, if the index is 1, then there is a stable distribution which is not a shift of a strictly stable distribution.

A distribution $\nu$ on $\boldsymbol{R}^{d}$ is said to be in the strict domain of attraction of a strictly stable $\mu$, if, for some $B_{n}>0$, the distribution of $S_{n}=B_{n}^{-1} \sum_{k=1}^{n} X_{k}$ converges to $\mu$, where $X_{1}, X_{2}, \cdots$ is a sequence of i.i.d. (independent and identically distributed) random vectors with distribution $\nu$. If the index is not 1, characterization of the strict domain of attraction is essentially known. However, the case of index 1 is quite different.

The first purpose of this paper is to find necessary and sufficient conditions for distributions to belong to the strict domain of attraction of a strictly stable law with index 1 , and the second purpose is to classify distributions in the strict domain of attraction according to their tail behavior.

Examples in the last section will illustrate remarkable difference between the case of index 1 and the other cases.

\section{§ 2. Preliminaries}

First we introduce some notations. Let $\boldsymbol{R}^{d}$ be the $d$-dimensional Euclidean space. We consider it as the set of column vectors with $d$ components with inner product $x y=\sum_{j=1}^{d} x_{j} y_{j}$ for $x=\left(x_{j}\right)_{1 \leq j \leq d}$ and $y=\left(y_{j}\right)_{1 \leq j \leq d}$ and with norm $|x|=(x x)^{1 / 2}$. Let $S^{d-1}$ be the unit sphere in $\boldsymbol{R}^{d}$. The $\sigma$-algebras of Borel subsets of $\boldsymbol{R}^{d}$ and of $S^{d-1}$ are denoted by $\mathscr{B}\left(\boldsymbol{R}^{d}\right)$ and $\mathscr{B}\left(S^{d-1}\right)$, respectively. The sets of all probability measures on $R^{d}$ and $S^{d-1}$ are denoted by $\mathscr{P}\left(\boldsymbol{R}^{d}\right)$ and $\mathscr{P}\left(S^{d-1}\right)$, respectively. For $\mu \in \mathscr{P}\left(\boldsymbol{R}^{d}\right)$, the characteristic function of $\mu$ is denoted by $\hat{\mu}$. For a random variable $X, \mu_{X}$ denotes 
the distribution of $X$. For $\mu, \nu \in \mathscr{P}\left(\boldsymbol{R}^{d}\right), \mu * \nu$ denotes the convolution of $\mu$ and $\nu$. We call a convolution of $\mu$ and a delta distribution a shift of $\mu$. For $\mu_{n}, \mu \in \mathscr{P}\left(\boldsymbol{R}^{d}\right), \mu_{n} \rightarrow \mu$ denotes weak convergence of $\mu_{n}$ to $\mu$. For $\nu \in \mathscr{P}\left(\boldsymbol{R}^{d}\right)$ and $A \in \mathscr{B}\left(\boldsymbol{R}^{d}\right), \int_{A} x \nu(d x) \operatorname{denotes}\left(\int_{A} x_{f} \nu(d x)\right)_{1 \leq j \leq d}$.

Definition 2.1. A distribution $\mu \in \mathscr{P}\left(\boldsymbol{R}^{d}\right)$ is strictly stable if for $a>0$, $b>0$, there exists $c>0$ such that

$$
\hat{\mu}(a z) \hat{\mu}(b z)=\hat{\mu}(c z), \quad z \in \boldsymbol{R}^{d} .
$$

It is well known that except in the case of $\delta_{0}$ (delta distribution at 0 ) this $c$ is uniquely determined by $a$ and $b$, and there exists $\alpha$ with $0<\alpha \leq 2$ such that $c^{\alpha}=a^{\alpha}+b^{\alpha}$. This $\alpha$ is called the index (exponent) of the strictly stable law. Distributions $\delta_{a}$ (delta distribution at $a$ ) with $a \neq 0$ are called trivial strictly stable distributions with index 1 .

The following theorem characterizes strictly stable laws as limit distributions. The proof can be given in a way similar to the case of stable laws.

THEOREM 2.2. A distribution $\mu$ on $\boldsymbol{R}^{d}$ is strictly stable if and only if there exist a sequence $X_{1}, X_{2}, \cdots, X_{n}, \cdots$ of i.i.d. random vectors and positive numbers $B_{n}$ such that $\mu_{S_{n}} \rightarrow \mu$, where

$$
S_{n}=B_{n}^{-1} \sum_{k=1}^{n} X_{k}
$$

Definition 2.3. Let $X_{1}, X_{2}, \cdots, X_{n}, \cdots$ be a sequence of i.i.d. random vectors with distribution $\nu$. If, for suitably chosen positive constants $B_{n}$, the distribution of (2.2) converges as $n \rightarrow \infty$ to a distribution $\mu$, then we say that $\nu$ is strictly attracted to $\mu$. We call the totality of distributions strictly attracted to $\mu$ the strict domain of attraction of $\mu$. Instead of (2.2), if, for suitably chosen constants $B_{n}$ and $A_{n} \in \boldsymbol{R}^{d}$, the distribution of $S_{n}=B_{n}^{-1} \sum_{k=1}^{n} X_{k}$ $-A_{n}$ converges as $n \rightarrow \infty$ to a distribution $\mu$, then we say that $\nu$ is attracted to $\mu$. As usual we call the totality of distributions attracted to $\mu$ the domain of attraction of $\mu$. Let $\boldsymbol{D}(\mu)$ and $\boldsymbol{S D}(\mu)$ denote the domain of attraction of $\mu$ and the strict domain of attraction of $\mu$, respectively.

From the above theorem, a distribution has a non-empty strict domain of attraction if and only if it is strictly stable. It is well known that a distribution has a non-empty domain of attraction if and only if it is stable. Characterization of the domain of attraction of a stable law is given in B.V. Gnedenko and A.N. Kolmogorov [3], I. A. Ibragimov and Yu. V. Linnik [4], W. Feller [2] and E.L. Rvačeva [6]. 
It is well known that if $\mu$ is strictly stable with index $\alpha$, then the normalizing constants $B_{n}$ in (2.2) is written as

$$
B_{n}=n^{1 / \alpha} h(n),
$$

where $h(x)$ is a slowly varying function, i.e. $h(x)$ is real-valued, positive and measurable on $[A, \infty)$ for some $A>0$, and, for each $k>0, \lim _{x \rightarrow \infty} h(k x) / h(x)$ $=1([1])$.

Definition 2.4. The slowly varying $h(x)$ appearing in (2.3) is called the slowly varying function part of normalizing constant $B_{n}$.

Definition 2.5. Distributions $\mu_{1}, \mu_{2} \in \mathscr{P}\left(\boldsymbol{R}^{d}\right)$ are strictly type-equivalent if there exists a constant $c>0$ such that $\hat{\mu}_{1}(z)=\hat{\mu}_{2}(c z)$.

Let $\mu_{1}$ and $\mu_{2}$ be strictly type-equivalent and $\hat{\mu}_{1}(z)=\hat{\mu}_{2}(c z)$. Obviously, their strict domain of attraction are identical. If $\nu$ is in this strict domain of attraction and if, for $i=1,2, B_{n, i}$ is the normalizing constant in (2.2) for $\mu_{i}$ and $h_{i}(x)$ is the slowly varying function in (2.3), then

$$
\lim _{x \rightarrow \infty} h_{1}(x) / h_{2}(x)=c .
$$

If $\mu_{1}$ and $\mu_{2}$ are not strictly type-equivalent, then $\boldsymbol{S D}\left(\mu_{1}\right) \cap \boldsymbol{S D}\left(\mu_{2}\right)=\varnothing$. These facts are proved by the convergence of types lemma ([3], Section 10). Hence it is possible to speak of the strict domain of attraction of a strict type. We say that slowly varying functions are equivalent if (2.4) holds for some $c(>0)$. Thus, with each $\nu$ in the strict domain of attraction, an equivalence class of slowly varying functions is associated.

\section{§3. Characterization of strict domain of attraction}

In this section, we give a necessary and sufficient conditions for distributions to belong to the strict domain of attraction of a strictly stable law with index 1 .

A canonical representation of strictly stable laws with index 1 is as follows. A distribution $\mu$ on $\boldsymbol{R}^{d}$ has characteristic function

$$
\hat{\mu}(z)=\exp \left\{i \gamma z+c^{\prime} \int_{S^{d-1}} \lambda(d \xi) \int_{0}^{\infty}\left(e^{i u \xi z}-1-i u \xi z\left(1+u^{2}\right)^{-1}\right) u^{-2} d u\right\}
$$

with $\gamma \in \boldsymbol{R}^{d}, c^{\prime}>0, \lambda \in \mathscr{P}\left(S^{d-1}\right)$ satisfying $\int_{S^{d-1}} \xi \lambda(d \xi)=0$, if and only if $\mu$ is a non-trivial strictly stable distribution with index 1 .

In what follow, we denote $x=|x| \xi$ for $x \in \boldsymbol{R}^{d} \backslash\{0\}$. 
THEOREM 3.1. Let $\mu$ be a strictly stable distribution on $\boldsymbol{R}^{d}$ with the characteristic function (3.1). In order that a distribution $\nu$ on $\boldsymbol{R}^{\boldsymbol{d}}$ belongs to $S D(\mu)$, it is necessary and sufficient that the following conditions hold:

$$
\begin{aligned}
& \lim _{R \rightarrow \infty} \nu\left(|x|>R, \xi \in E_{1}\right) / \nu\left(|x|>R, \xi \in E_{2}\right)=\lambda\left(E_{1}\right) / \lambda\left(E_{2}\right), \\
& \quad \text { for all } \lambda \text {-continuous sets } E_{1}, E_{2} \in \mathscr{B}\left(S^{d-1}\right) . \\
& \lim _{R \rightarrow \infty} \nu(|x|>R) / \nu(|x|>k R)=k \quad \text { for all } k>0 . \\
& \lim _{R \rightarrow \infty} \int_{|x|<R} x \nu(d x) / R \nu(|x|>R)=\gamma / c^{\prime} .
\end{aligned}
$$

REMARK. The conditions (3.2) and (3.3) together are equivalent to the condition that for all $\lambda$-continuous sets $E_{1}, E_{2} \in \mathscr{B}\left(S^{d-1}\right)$ and $k>0$,

$$
\lim _{R \rightarrow \infty} \nu\left(|x|>R, \xi \in E_{1}\right) / \nu\left(|x|>k R, \xi \in E_{2}\right)=k \lambda\left(E_{1}\right) / \lambda\left(E_{2}\right) .
$$

If we drop the restriction $\int_{S^{d-1}} \xi \lambda(d \xi)=0$, then (3.1) is the canonical representation of a stable law with index 1 and the domain of attraction of the law in question is characterized by (3.5).

If, in particular, $d=1$, the above theorem takes the following form.

Theorem 3.2. Let $\mu$ be a Cauchy distribution with characteristic function

$$
\hat{\mu}(z)=\exp (i \gamma z-c|z|) \quad \text { where } \gamma \in \boldsymbol{R}^{1}, c>0 .
$$

In order that a distribution $\nu$ on $\boldsymbol{R}^{1}$ belong to $\boldsymbol{S D}(\mu)$, it is necessary and sufficient that

$$
\begin{aligned}
& \lim _{x \rightarrow \infty} \nu(-\infty,-x] / \nu[x, \infty)=1, \\
& \lim _{x \rightarrow \infty}\{\nu(-\infty,-x]+\nu[x, \infty)\} /\{\nu(-\infty,-k x]+\nu[k x, \infty)\}=k \quad \text { for all } k>0, \\
& \lim _{x \rightarrow \infty} \int_{|t|<x} t \nu(d t) / x \nu[x, \infty)=\pi \gamma / c .
\end{aligned}
$$

Proof of Theorem 3.1. By E.L. Rvačeva [6], it is sufficient to show that (3.2)-(3.4) are equivalent to the fact that there exist constants $B_{n}>0$ satisfying the following (3.10)-(3.12).

$$
\begin{aligned}
& \lim _{n \rightarrow \infty} n \nu\left(|x|>R B_{n}, \xi \in E\right)=c^{\prime} \lambda(E) / R \\
& \quad \text { for any } \lambda \text {-continuous set } E \in \mathscr{B}\left(S^{d-1}\right) \text { and } R>0 .
\end{aligned}
$$

$$
\lim _{\varepsilon \rightarrow 0} \varlimsup_{n \rightarrow \infty} n B_{n}^{-2}\left[\int_{|x|<\varepsilon B_{n}}(z x)^{2} \nu(d x)-\left\{\int_{|x|<\& B_{n}} z x \nu(d x)\right\}^{2}\right]=0 \quad \text { for } z \in R^{d} .
$$




$$
\lim _{n \rightarrow \infty} n B_{n}^{-1} \int_{|x|<\tau B_{n}} x \nu(d x)=\gamma \quad \text { for } \tau>0
$$

It can be proved in a similar manner to E.L. Rvačeva [6] that the conditions (3.2) and (3.3) are equivalent to the existence of $B_{n}>0$ satisfying (3.10) and (3.11). Hence, we have to deal only with the last condition. It is easy to see that (3.2)-(3.4) imply (3.12), because we get

$$
\begin{aligned}
\lim _{n \rightarrow \infty} n B_{n}^{-1} \int_{|x|<\tau B_{n}} x \nu(d x) & =\lim _{n \rightarrow \infty} n B_{n}^{-1} \gamma c^{\prime-1} \tau B_{n} \nu\left(|x|>B_{n} \tau\right) \\
& =\gamma c^{\prime-1} \lim _{n \rightarrow \infty} n \tau \nu\left(|x|>B_{n} \tau\right)=\gamma,
\end{aligned}
$$

using (3.10). Next we prove that (3.10)-(3.12) imply (3.4). From (3.10) and (3.12),

$$
\lim _{n \rightarrow \infty} n B_{n}^{-1} \int_{|x|<B_{n} R} x \nu(d x) / R n \nu\left(|x|>B_{n} R\right)=\gamma / c^{\prime} .
$$

Hence it is enough to show that

$$
\lim _{R \rightarrow \infty} \int_{|x|<R} x \nu(d x) / R \nu(|x|>R)=\lim _{n \rightarrow \infty} \int_{|x|<B_{n}} x \nu(d x) / B_{n} \nu\left(|x|>B_{n}\right) .
$$

Since we can prove that $\tilde{B}_{n}=\max _{1 \leq k \leq n} B_{k}$ are also normalizing constants ([1], p. 23: monotone equivalents of regularly varying function), we assume that $B_{n}$ is an increasing sequence. Let

$$
V_{n}=\max _{B_{n} \leq R \leq B_{n+1}}\left|\int_{|x|<R} x_{j} \nu(d x) / R \mathcal{L}(|x|>R)-\int_{|x|<B_{n}} x_{j} \nu(d x) / B_{n} \nu\left(|x|>B_{n}\right)\right| .
$$

We will prove that $\lim _{n \rightarrow \infty} V_{n}=0$. It is noted that

$$
\int_{|x|<R} x_{j} \nu(d x) / R \nu(|x|>R)-\int_{|x|<B_{n}} x_{j} \nu(d x) / B_{n} \nu\left(|x|>B_{n}\right)=f(n, R)+g(n, R),
$$

where $f(n, R)=\left(\int_{|x|<R} x_{j} \nu(d x)-\int_{|x|<B_{n}} x_{j} \nu(d x)\right) / R \nu(|x|>R)$,

$$
g(n, R)=\left\{1-R \nu(|x|>R) / B_{n} \nu\left(|x|>B_{n}\right)\right\} \int_{|x|<B_{n}} x_{j} \nu(d x) / R \nu(|x|>R) .
$$

Now set

Since

$$
V_{n}^{1}=\max _{B_{n} \leq R \leq B_{n+1}}|f(n, R)| \text { and } V_{n}^{2}=\max _{B_{n} \leq R \leq B_{n+1}}|g(n, R)|
$$

$$
\begin{aligned}
& V_{n}^{1}=\max _{B_{n} \leq R \leq B_{n+1}}\left|\int_{B_{n} \leq|x|<R} x_{j} \nu(d x)\right| / R \nu(|x|>R) \\
& \quad \leq \max _{B_{n} \leq R \leq B_{n+1}} \nu\left(|x| \geq B_{n}\right) / \nu(|x|>R)-1,
\end{aligned}
$$


we see that $\lim _{n \rightarrow \infty} V_{n}^{1}=0$. Here we have used the facts that $R \nu(|x|>R)$ is slowly varying and that $B_{n+1} / B_{n} \rightarrow 1$ as $n \rightarrow \infty$. By the same reason, we have

$$
\begin{aligned}
& \lim _{n \rightarrow \infty} \max _{B_{n} \leq R \leq B_{n+1}}\left|R \nu(|x|>R) / B_{n} \nu\left(|x|>B_{n}\right)-1\right|=0 \text { and } \\
& \lim _{n \rightarrow \infty} \max _{B_{n} \leq R \leq B_{n+1}}\left|\int_{|x|<B_{n}} x_{j} \nu(d x) / R \nu(|x|>R)-\gamma_{j} / c^{\prime}\right|=0,
\end{aligned}
$$

which imply $\lim _{n \rightarrow \infty} V_{n}^{2}=0$. Therefore $\lim _{n \rightarrow \infty} V_{n}=0$, which proves (3.13). Hence the proof is complete.

In case $\alpha \neq 1$, strict domains of attraction are described by the following theorems: Theorems 3.3-3.5.

Theorem 3.3. Assume that $0<\alpha<1$. A distribution $\mu$ on $\boldsymbol{R}^{d}$ is strictly stable with index $\alpha$ if and only if

$$
\hat{\mu}(z)=\exp \left\{c^{\prime} \int_{S^{d-1}} \lambda(d \xi) \int_{0}^{\infty}\left(e^{i u \xi z}-1\right) u^{-1-\alpha} d u\right\}
$$

with $c^{\prime}>0, \lambda \in \mathscr{P}\left(S^{d-1}\right)$. Let $\mu$ be strictly stable with index $\alpha$ having characteristic function (3.14). Then $\nu \in S \boldsymbol{D}(\mu)$ if and only if $\nu$ satisfies (3.2) and (3.3) with $k$ in the right-hand side replaced by $k^{\alpha}$.

Theorem 3.4. i) Assume that $1<\alpha<2$. A distribution $\mu$ on $\boldsymbol{R}^{d}$ is strictly stable with index $\alpha$ if and only if

$$
\hat{\mu}(z)=\exp \left\{c^{\prime} \int_{S^{d-1}} \lambda(d \xi) \int_{0}^{\infty}\left(e^{i u \xi z}-1-i u \xi z\right) u^{-1-\alpha} d u\right\}
$$

with $c^{\prime}>0, \lambda \in \mathscr{P}\left(S^{d-1}\right)$.

ii) Let $\mu$ be a distribution with the characteristic function (3.15). Then $\nu \in \boldsymbol{S D}(\mu)$ if and only if $\nu$ has mean 0 and satisfies (3.2) and (3.3) with $k$ in the right-hand side replaced by $k^{\alpha}$.

THEOREM 3.5. A distribution $\mu$ on $\boldsymbol{R}^{d}$ is strictly stable with index 2 if and only if $\mu$ is Gaussian (possibly degenerate) with mean 0 and $\mu \neq \delta_{0}$. Let $\mu$ be strictly stable with index 2 and let $\left(a_{j k}\right)$ be its covariance matrix. Set $A(z)=\sum_{j, k=1}^{d} a_{j k} z_{j} z_{k}$. Then $\nu \in S D(\mu)$ if and only if it satisfies the following three conditions:

$$
\begin{aligned}
& \lim _{R \rightarrow \infty} R^{2} \nu(|x|>R) / \int_{|x|<R}|x|^{2} \nu(d x)=0 \\
& \lim _{R \rightarrow \infty} \int_{|x|<R}(z x)^{2} \nu(d x) / \int_{|x|<R}(w x)^{2} \nu(d x)=A(z) / A(w),
\end{aligned}
$$

where $z, w \in \boldsymbol{R}^{d}$ with $A(w) \neq 0$, and where 


$$
\int_{R^{d}} x \nu(d x)=0
$$

The canonical form of characteristic functions in Theorems 3.1-3.5 are given in [5] and [8]. The proof of the characterization of the strict domain of attraction in Theorems $3.3-3.5$ can be given in a similar manner to the proof of Theorem 3.1 if we make use of properties of regularly varying functions in Theorems 1 and 2 in [2], Chapter 8 Section 9.

\section{§ 4. Classification of the strict domain of attraction}

In this section, we show some properties of distributions in the strict domain of attraction. Having observed the difference between the domain of attraction and the strict domain of attraction, we have the following two propositions in the case of $\alpha \neq 1$. They are consequences of Theorems 3.3-3.5.

Proposition 4.1. Let $\nu$ be a distribution in the domain of attraction of a stable law $\mu$ with index $0<\alpha<1$. Then $\nu$ belongs to the strict domain of attraction of a strictly stable law $\mu_{s}=\mu * \delta_{s}$ with some $s \in \boldsymbol{R}^{d}$. More precisely, if $X_{1}, X_{2}, \ldots$ is a sequence of i.i.d. random vectors with distribution $\nu$ and the distribution of

$$
S_{n}=B_{n}^{-1} \sum_{k=1}^{n} X_{k}-A_{n}
$$

converges to $\mu$, then the distribution of $B_{n}^{-1} \sum_{k=1}^{n} X_{k}$ converges to $\mu_{s}$ and the sequence $A_{n}$ converges to $s$.

Proposition 4.2. Let $\nu$ be a distribution in the domain of attraction of a stable law with index $1<\alpha \leq 2$. Then $\nu$ belongs to the strict domain of attraction of a strictly stable law if and only if $\nu$ has mean 0.

Propositions 4.1 and 4.2 imply that if $\nu$ lies in the domain of attraction of a stable law with index $\alpha \neq 1$, then $\nu$ itself or a shift of $\nu$ belongs to the strict domain of attraction of some strictly stable law. However, the case of index 1 is more delicate and the total behavior of the distribution is relevant.

The main results in this section start with the assertion that any slowly varying function can appear in the slowly varying function part of the normalizing constants for distributions in the strict domain of attraction of strictly stable distributions. For $d=1$, H.G. Tucker [10] shows a similar fact for the (non-strict) domain of attraction. But in the case of the strict domain of attraction for index 1 we have to make full use of Theorem 3.1. 
TheOREm 4.3. Let $0<\alpha<2$. For any non-trivial strictly stable law $\mu$ with index $\alpha$ and any slowly varying function $h(x)$, there exists a distribution $\nu$ on $\boldsymbol{R}^{d}$ such that $\mu_{S_{n}} \rightarrow \mu$, where $S_{n}=\left(n^{1 / \alpha} h(n)\right)^{-1} \sum_{k=1}^{n} X_{k}$ and $X_{k}$ is a sequence of i.i.d. random vectors with distribution $\nu$.

Proof. We prove the assertion in the case of $\alpha=1$ in detail. A distribution $\nu$ belongs to the domain of attraction of the strictly stable law $\mu$ with characteristic function (3.1) if and only if there is a slowly varying function $H(R)$ such that

$$
\begin{aligned}
& \nu(|x|>R, \xi \in E)=c^{\prime}(\lambda(E)+o(1)) H(R) R^{-1} \\
& \quad \text { for each } \lambda \text {-continuous set } E \in \mathscr{P}\left(S^{d-1}\right),
\end{aligned}
$$

where $o(1)$ is a term which tends to 0 as $R \rightarrow \infty$. If $\nu$ satisfies this condition, then $B_{n}=n^{1 / \alpha} h(n)$ is the normalizing constant for $\nu$ if and only if $h(R)$ and $H(R)$ satisfy the relation

$$
\lim _{R \rightarrow \infty} H(R h(R)) / h(R)=1 .
$$

We are now in a position to construct such a distribution $\nu$ and a function $H(R)$ satisfying (4.2), (4.3) and (3.4) for given $h(x)$. It follows from the existence theorem of asymptotic inverses of regularly varying functions ([7], p. 23) that, for each given $h(x)$, there exists a slowly varying function $H(R)$ satisfying (4.3). By the representation theorem of slowly varying function ([7], p. 2), $H(R)$ on $[A, \infty)$ is written as

$$
H(R)=c(R) \exp \int_{B}^{R} \varepsilon(t) t^{-1} d t, \quad R \geq A
$$

where $\lim _{R \rightarrow \infty} c(R)=c(0<c<\infty), B$ is a positive constant and $\varepsilon(t)$ is a continuous function such that $\lim _{t \rightarrow \infty} \varepsilon(t)=0$. In our situation we can choose an appropriate $H(R)$ satisfying (4.3) such that $\varepsilon(t) \leq 1, c(R)$ is a positive constant and $A=B=1$. Thus $H^{\prime}(R)=H(R) \varepsilon(R) R^{-1}$. Define $\varepsilon_{+}(u), \varepsilon_{-}(u)$ by $\varepsilon_{+}(u)=\varepsilon(u) \vee 0, \varepsilon_{-}(u)=-(\varepsilon(u) \wedge 0)$. We have $\varepsilon(u)=\varepsilon_{+}(u)-\varepsilon_{-}(u)$. Let $e_{i}$ denote the unit vector with $i$-th component 1 . With the $e_{i}$, we define a measure $\sigma$ on $S^{d-1}$ in the following way: Support of $\sigma=\left\{ \pm e_{i}: i=1,2, \cdots, d\right\}$ and $\sigma\left(\left\{e_{i}\right\}\right)=\sigma\left(\left\{-e_{i}\right\}\right)=\left|\gamma_{i}\right| c^{\prime-1}$. On the interval $(1, \infty)$, define measures $\rho_{e_{i}}$ and $\rho_{-e_{\boldsymbol{t}}}, i=1,2, \cdots, d$, as follows:

Then

$$
\begin{array}{ll}
\rho_{ \pm e_{i}}(d u)=\varepsilon_{ \pm}(u) d u & \text { if } \gamma_{i} \geq 0, \\
\rho_{ \pm e_{i}}(d u)=\varepsilon_{\mp}(u) d u & \text { if } \gamma_{i}<0 .
\end{array}
$$

$$
\int_{S^{d-1}} \xi_{j} \sigma(d \xi) \rho_{\xi}(d u)=\gamma_{j} c^{\prime-1} \varepsilon(u) d u
$$


We define a finite measure $\nu_{0}$ on $R^{d}$ by

$$
\begin{aligned}
\nu_{0}(E)= & \sum_{i=1}^{d}\left|\gamma_{i}\right| H(1) 1_{E}\left(\left(\operatorname{sgn} \gamma_{i}\right) e_{i}\right) \\
& +c^{\prime}\left\{\int_{S^{d-1}} \lambda(d \xi) \int_{1}^{\infty} 1_{E}(u \xi)(1-\xi(u)) H(u) u^{-2} d u\right. \\
& \left.+\int_{S^{d-1}} \sigma(d \xi) \int_{1}^{\infty} 1_{E}(u \xi) H(u) u^{-2} \rho_{\xi}(d u)\right\} \quad \text { for } E \in \mathscr{B}\left(\boldsymbol{R}^{d}\right),
\end{aligned}
$$

where $1_{E}$ is the indicator function of $E$. Define a probability measure $\nu_{1}=$ $c_{0}^{-1} \nu_{0}$, where $c_{0}$ is the total measure of $\nu_{0}$.

Then, we verify that $\nu_{1}$ satisfies an analogue of (4.2). If $R>1$, then

$$
\begin{aligned}
\nu_{1}(|x|> & R, \xi \in E) \\
& =c_{0}^{-1} c^{\prime}\left\{\lambda(E) \int_{R}^{\infty}(1-\varepsilon(u)) H(u) u^{-2} d u+\int_{E} \sigma(d \xi) \int_{R}^{\infty} H(u) u^{-2} \rho_{\xi}(d u)\right\} \\
& =c_{0}^{-1} c^{\prime}\left\{\lambda(E) H(R) R^{-1}+\int_{E} \sigma(d \xi) \int_{R}^{\infty} H(u) u^{-2} \rho_{\xi}(d u)\right\} \\
& =c_{0}^{-1} c^{\prime}\left\{\lambda(E)+R H(R)^{-1} \int_{E} \sigma(d \xi) \int_{R}^{\infty} H(u) u^{-2} \rho_{\xi}(d u)\right\} H(R) R^{-1}
\end{aligned}
$$

Since

$$
R H(R)^{-1} \int_{E} \sigma(d \xi) \int_{R}^{\infty} H(u) u^{-2} \rho_{\xi}(d u) \leq 2 \sum_{j=1}^{d}\left|\gamma_{j}\right| c^{\prime-1} R H(R)^{-1} \int_{R}^{\infty} H(u) u^{-2}|\varepsilon(u)| d u
$$

we have

$$
\lim _{R \rightarrow \infty} R H(R)^{-1} \int_{E} \sigma(d \xi) \int_{R}^{\infty} H(u) u^{-2} \rho_{\xi}(d u)=0 .
$$

Hence $\nu_{1}$ satisfies (4.2) with $c^{\prime}$ replaced by $c_{0}^{-1} c^{\prime}$.

Next we prove that $\nu_{1}$ satisfies (3.4). We have

$$
\int_{|x|<R} x_{j} \nu_{1}(d x)=\int_{|x| \leq 1} x_{j} \nu_{1}(d x)+\int_{1<|x|<R} x_{j} \nu_{1}(d x)
$$

the first term of which is equal to $c_{0}^{-1} \gamma_{j} H(1)$. Noticing $\int_{S^{a-1}} \xi_{j} \lambda(d \xi)=0$ and usign (4.6) we see that the second term equals

$$
c_{0}^{-1} \gamma_{j} \int_{1}^{R} H(u) u^{-1} \varepsilon(u) d u=c_{0}^{-1} \gamma_{j}(H(R)-H(1)) .
$$

Therefore $\int_{|x|<R} x_{j} \nu_{1}(d x)=c_{0}^{-1} \gamma_{j} H(R)$. Since $\quad R \nu_{1}(|x|>R)=c_{0}^{-1} c^{\prime} H(R)(1+$ $o(1)$ ), the equation (3.4) is proved. 
Now we conclude that $\nu_{1}$ belongs to the strict domain of attraction of $\mu$ with normalizing constant $c_{0}^{-1} n h(n)$. Define $\nu$ by $\nu(E)=\nu_{1}\left(c_{0}^{-1} E\right)$. Then $\nu$ is a distribution satisfying the desired conditions.

In the case $\alpha \neq 1$, for any given slowly varying function $h(x)$, we can construct a distribution $\nu$ with an appropriate tail behavior in a similar way to the case of $\alpha=1$. In the case $0<\alpha<1$, this $\nu$ is a distribution in $\boldsymbol{S D}(\mu)$ having the desired property by Proposition 4.1. In the case $1<\alpha<2$, the shifted measure $\nu * \delta_{-m}$ ( $m$ is the mean of $\nu$ ) is the desired distribution by Proposition 4.2. Thus the proof is complete.

REMARK. In the case of index 2, the above theorem does not hold. In fact, let $\mu$ be a Gaussian distribution (possibly degenerate) with mean 0 and $\mu \neq \delta_{0}$. Then for some $\nu \in \boldsymbol{S D}(\mu)$ a slowly varying function $h(x)$ is the slowly varying function part of normalizing constant for $\nu$ if and only if

$$
\lim _{x \rightarrow \infty} \max _{t \leq x} h(t) / h(x)=1 .
$$

This condition means that $h(x)$ is equivalent with an increasing slowly varying function. (This fact is proved for $d=1$ in [10] and for general $d$ in [9]).

Next we study the problem whether shifts of a distribution in a strict domain of attraction belong to a strict domain of attraction. In this consideration the slowly varying function part of normalizing constant plays an important role. For a non-trivial strictly stable law $\mu$ with index 1 on $\boldsymbol{R}^{d}$, we classify the strict domain of attraction of a strictly stable law with index 1 on $\boldsymbol{R}^{d}$ as follows.

$$
\begin{aligned}
& \boldsymbol{S D}_{1}(\mu)=\left\{\nu \in \boldsymbol{S D}(\mu): \lim _{R \rightarrow \infty} R \nu(|x|>R)=\infty\right\} . \\
& \boldsymbol{S D}_{2}(\mu)=\left\{\nu \in \boldsymbol{S D}(\mu): \lim _{R \rightarrow \infty} R \nu(|x|>R)=c(0<c<\infty)\right\} . \\
& \boldsymbol{S D}_{3}(\mu)=\left\{\nu \in \boldsymbol{S D}(\mu): \lim _{R \rightarrow \infty} R \nu(|x|>R)=0 \text { or the limit does not exist }\right\} .
\end{aligned}
$$

For $\nu \in \mathscr{P}\left(\boldsymbol{R}^{d}\right)$ and $s \in \boldsymbol{R}^{d}$, let $\nu_{s}$ denote $\nu * \delta_{s}$.

THEOREM 4.4. Each $\boldsymbol{S D}_{i}(\mu)(i=1,2,3)$ is non-empty. If $\nu \in \boldsymbol{S D}_{1}(\mu)$, then, for all $s \in \boldsymbol{R}^{d}, \nu_{s} \in \boldsymbol{S} \boldsymbol{D}_{1}(\mu)$. If $\nu \in \boldsymbol{S D}_{2}(\mu)$, then, for each $s \in \boldsymbol{R}^{a}, \nu_{s} \in \boldsymbol{S} \boldsymbol{D}\left(\mu_{m(s)}\right)$ for $m(s)=c^{-1} s$ where $c=\lim _{R \rightarrow \infty} R \nu(|x|>R)$. If $\nu \in S D_{3}(\mu)$, then, for every $s \in \boldsymbol{R}^{d} \backslash\{0\}$ and every strictly stable law $\tilde{\mu}, \nu_{s} \notin \boldsymbol{S D}(\tilde{\mu})$.

Proof. The classification is made, based on the slowly varying function $H(R)$ appearing in (4.2). Namely, $R \nu(|x|>R)$ is a slowly varying function equivalent to $H(R)$. Hence it follows from (4.3) that $\nu \in S D_{1}(\mu)\left(S D_{2}(\mu)\right.$, 
$\boldsymbol{S D}_{3}(\mu)$ ) if and only if the slowly varying function $h(R)$ associated with $\nu$ tends to $\infty$ (to $c(0<c<\infty)$, to 0 or the limit does not exist). Therefore all the cases are possible by Theorem 4.3. Let $\nu$ be in the strict domain of attraction of the strictly stable law with characteristic function (3.1). We have

$$
\begin{aligned}
\int_{|x|<R} x_{j} \nu_{s}(d x) / R \nu_{s}(|x|>R)= & \left\{\int_{|x|<R} x_{j} \nu(d x) / R \nu(|x|>R)\right\}\left(1+f_{1}(R)\right) \\
& +s_{j}\{R \nu(|x|>R)\}^{-1}\left(1+f_{2}(R)\right)+f_{3}(R)
\end{aligned}
$$

where

$$
\begin{aligned}
& f_{1}(R)=\nu(|x|>R) / \nu_{s}(|x|>R)-1, \\
& f_{2}(R)=\left\{\nu(|x|>R) / \nu_{s}(|x|>R) \nu_{s}(|x|<R)-1,\right. \\
& f_{3}(R)=\left\{R \nu_{s}(|x|>R)\right\}^{-1}\left(\int_{|x+s|<R} x_{j} \nu(d x)-\int_{|x|<R} x_{j} \nu(d x)\right) .
\end{aligned}
$$

We can prove $\lim _{R \rightarrow \infty} f_{i}(R)=0 i=1,2,3$ by calculation based on the fact that $R \nu(|x|>R)$ is slowly varying. Since $\nu_{s}$ automatically satisfies (3.2) and (3.3), the rest of the proof follows from the equality (4.9).

REMARK. We can call $\boldsymbol{S D}_{2}(\mu)$ the strict domain of normal attraction. In this case, let $\mu_{1}$ and $\mu_{2}$ be type-equivalent strictly stable laws with index 1 . Then, for any $\nu \in \boldsymbol{S D}_{2}\left(\mu_{1}\right)$, there exists $s \in \boldsymbol{R}^{d}$ uniquely such that $\nu_{s} \in \boldsymbol{S D}_{2}\left(\mu_{2}\right)$.

REMARK. Let $\mu$ be a stable law with index $\alpha \neq 1$. In case $0<\alpha<1$, if $\nu \in \boldsymbol{S} \boldsymbol{D}(\mu)$, then $\nu_{s} \in \boldsymbol{S} \boldsymbol{D}(\mu)$ for each $s \in \boldsymbol{R}^{d}$. In case $1<\alpha \leq 2$, if $\nu \in \boldsymbol{S} \boldsymbol{D}(\mu)$ and if $\tilde{\mu}$ is a strictly stable law, then $\nu_{s} \notin \boldsymbol{S} \boldsymbol{D}(\tilde{\mu})$ for every $s \notin \boldsymbol{R}^{d} \backslash\{0\}$.

\section{§ 5. Examples}

There are some more interesting properties that are worth mentioning regarding the strict domain of attraction with index 1 . Two examples in this section will serve to explain this situation. We fix a strictly stable law $\mu$ with index 1 on $\boldsymbol{R}^{1}$ and consider the difference between $\boldsymbol{D}(\mu)$ and $\boldsymbol{S D}(\mu)$. Example 1 implies that the class of all shifts of distributions in $\boldsymbol{S D}(\mu)$ is a proper subclass of $\boldsymbol{D}(\mu)$. Example 2 shows that a distribution in $\boldsymbol{D}(\mu)$ with mean 0 does not necessarily belong to $\boldsymbol{S D}(\mu)$. These properties are peculiar to the case of index 1 .

EXAMPLE 1. Let $\nu$ be a distribution with the following density function:

$$
p(x)= \begin{cases}c_{0} x^{-2}\left(1+(\log x)^{-1}\right) & x \geq e \\ 0 & |x|<e \\ c_{0} x^{-2} & x \leq-e\end{cases}
$$


where $c_{0}$ is a normalizing constant. It is easy to prove that $\nu$ belongs to the domain of attraction of Cauchy distribution. For each $s \in \boldsymbol{R}^{\mathbf{1}}$, we can prove

$$
\begin{aligned}
\int_{|t|<x} t \nu_{s}(d t) / x \nu_{s}[x, \infty) & =(1+o(1))\left[\log ^{2}(x-s)\right. \\
& \left.-\log \{(x+s) /(x-s)\}+s \nu_{s}(-x, x)\right] \quad \text { as } x \rightarrow \infty .
\end{aligned}
$$

This shows that, for each $s \neq 0, \nu_{s}$ does not belong to the strict domain of attraction of Cauchy distribution.

EXAMPLe 2. Let $\nu_{0}$ be a distribution with the density function

$$
p_{0}(x)= \begin{cases}c_{0}(x \log x)^{-2}(1+f(x)) & x \geq e \\ 0 & |x|<e \\ c_{0}(|x| \log |x|)^{-2} & x \leq-e\end{cases}
$$

where $f(x)$ is a continuous function on $[e, \infty)$ such that $f(x) \geq-1$ and $\lim _{x \rightarrow \infty} f(x)=0$ and $c_{0}$ is a normalizing constant. It is easy to see that $\nu_{0}$ belongs to the domain of attraction of Cauchy distribution and has finite mean $m$.

Let $\nu$ be $\nu_{0} * \delta_{-m}$. Obviously, $\nu$ belongs to the domain of attraction and has mean 0 . And we can prove the following equality if the limit in the right hand side exists:

$$
\lim _{x \rightarrow \infty} \int_{|t|<x} t \nu(d t) / x \nu[x, \infty)=-2^{-1} \lim _{x \rightarrow \infty} f(x) \log x .
$$

Therefore, if we set $f(x)=-2 \pi \gamma(c \log x)^{-1}(x \geq \exp (2 \pi \gamma / c \vee 0))$, then (3.9) is satisfied. But, if $f(x)=(\log x)^{-1 / 2}$, then (3.9) is not satisfied. This means that the condition that a distribution belongs to the domain of attraction and has mean 0 does not guarantee that it belongs to the strict domain of attraction.

Acknowledgement The author wishes to express his gratitude to Professor Ken-iti Sato for his guidance and encouragement in the course of the research.

\section{References}

[1] N. H. Bingham, C. M. Goldie, and J. L. Teugels, Regular Variation, Encyclopedia of Math. and Its Appl., 1987.

[2] W. Feller, An Introduction to Probability Theory and Its Applications, 2nd ed., Vol. II, Wiley, New York, 1971.

[ 3 ] B. V. Gnedenko and A. N. Kolmogorov, Limit Distributions for Sums of Independent Random Variables. (English translation) 2nd ed. Addison-Wesley, Cambridge, Mass, 1968. 
[4] I. A. Ibragimov and Yu. V. Linnik, Independent and Stationary Sequences of Random Variables, Wolters-Noordhoff Publishing, Groningen, The Netherlands, 1971.

[5] P. Lévy, Théorie de l'Addition des Variables Aléatoires. 2̇̀me éd. GauthierVillars, Paris, 1954 (1̀̀re éd., 1937).

[6] E. L. Rvačeva, On domain of attraction of multi-dimensional distributions, (English translation) Select. Transl. Math. Statist. and Probability, 2 (1954), 183205. (L'vov. Gos. Univ. Uč. Zap. 29 Ser, Meh.-Mat. No. 6, 5-44).

[7] E. Seneta, Regularly Varying Functions, Lecture Notes in Mathematics 508, Springer-Verlag, Berlin, 1976.

[8] K. Sato, Infinitely Divisible Distributions, Seminar on Probability, 52, Kakuritsuron Seminar (in Japanese), 1981.

[9] T. Shimura, Decomposition of non-decreasing slowly varying functions and the domain of attraction of Gaussian distributions, submitted to J. Math. Soc. Japan.

[10] H. G. Tucker, Convolutions of distributions attracted to stable laws, Ann. Math. Statist., 39 (5) (1968), 1381-1390.

\author{
DePaRTMENT OF MATHEMATICS \\ SCHOOL OF SCIENCE \\ Nagoya UNiversity \\ Chikusa-kU, Nagoya, 464 \\ JAPAN
}

